\title{
Vildagliptin Treatment on the Portal Venous Pressure and Hepatosteatosis in Patients with Type 2 Diabetes Mellitus
}

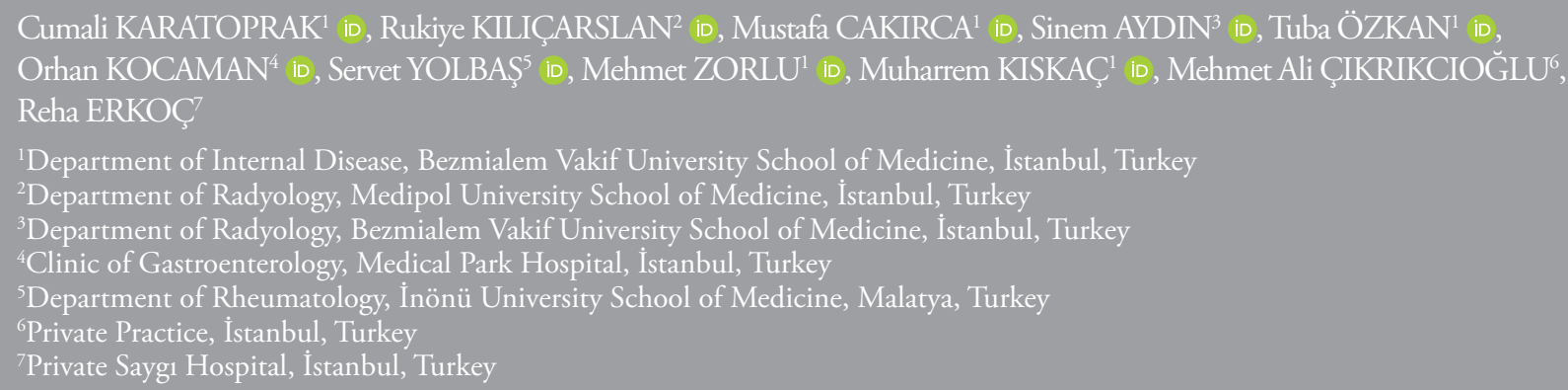

\section{ABSTRACT}

Objective: This study investigated how vildagliptin (a di-peptidyl peptidase 4 inhibitor) affects portal vein pressure and hepatosteatosis in patients with type 2 diabetes mellitus.

Methods: This cross-sectional study evaluated the use of specific drugs for at least 3 months on two groups of type 2 diabetes mellitus cases. Group 1 used metformin and gliclazide, Group 2 used the same amounts of metformin and gliclazide, with the addition of vildagliptin. Using Doppler ultrasound, all cases were measured for portal vein flow velocity, portal vein flow and portal vein diameter. Degree of hepatosteatosis was also recorded.

Results: A total of 97 patients completed the study. The study finished with 49 type 2 DM patients in Group1 (20 men, 29 women) and 48 patients in Group2 ( 20 men, 28 women. No significant difference was found in term of age, gender, BMI, HbA1c, mean arterial pressure, LDL-C, HDL-C or triglyceride levels in two groups.Portal vein flow velocity, portal vein flow volume, and portal vein diameter of all cases were measured by Doppler ultrasound in both groups. No significant difference was found between the groups (respectively $\mathrm{p}=0.92, \mathrm{p}=0.60, \mathrm{p}=0.92$ ). There was no significant difference between groups regarding to ultrasonographic grading of hepatosteatosis.

Conclusion: Treating type 2 diabetes mellitus patients with vildagliptin for had no effect on portal vein hemodynamics and hepatosteatosis as assessed with Doppler ultrasound. Further long-term studies with better evaluation methods are needed to demonstrate any expected beneficial effect of vildagliptin on portal hemodynamics and hepatosteatosis.

Keywords: Di-Peptidyl peptidase 4 inhibitors, vildagliptin, portal vein pressure, hepatosteatosis, type 2 diabetes mellitus

\section{Introduction}

Recent years have seen the development of drugs that increase plasma incretins for treatment of type 2 diabetes mellitus (DM). Incretins are secreted as an intestinal hormone by entero-endocrine cells immediately after meals for the purpose of regulating glucose. The two known incretins are glucose-dependent insulinotropic peptide (GIP) and glucagon-like peptide-1 (GLP-1). Both of the incretins are rapidly inactivated by the enzyme named dipeptil peptidase 4 (DPP-4). Developed DPP-4 inhibitor drugs increase the plasma concentrations of GIP and GLP-1 by preventing their degradation by inhibiting the pertinent enzyme $(1,2)$.

Although DPP-4 inhibitors mainly affect the pancreatic gland, they also affect the gastrointestinal tract, central nervous system, bone, adipose tissue, and the cardiovascular system $(1,2)$. They both incretins reduce intestinal motility, extend the

Cite this article as: Karatoprak C, Kılıçarslan R, Cakırca M, Aydın S, Özkan T, Kocaman O, et al. Vildagliptin Treatment on the Portal Venous Pressure and Hepatosteatosis in Patients with Type 2 Diabetes Mellitus. Bezmialem Science 2018; 6: 1-5. 
time of gastric emptying, and suppress gastric acid secretion, particularly in the gastrointestinal tract $(3,4)$. These effects may cause slight to severe nausea, vomiting, or bloating.

A previous study on dog fetus cell culture found that production of nitric oxide (NO) increased due to the incretin GIP, which in turn resulted in increased portal venous flow (5). $\mathrm{NO}$ is a potent short-lived vasodilatatory radical that plays an important role in the regulation of vascular tone (6). Increased NO secretion is one of the main responsible mediators that occurred from splanchnic vein hyperemia and vasodilatation of the portal vein.

In recent years, it was shown that ischemic injury plays an important role in the etiology of non-alcoholic fatty liver disease (NAFLD), the specific liver pathology of metabolic syndrome $(7,8)$. As research continues for definitive treatment, current treatment of fatty liver disease is directed against etiological subgroups such as obesity, hypertension, hyperlipidemia, and type 2 diabetes mellitus. Treatments that demonstrate efficacy in the treatment of type $2 \mathrm{DM}$ and in prevention of ischemic injury in the liver may be a novel treatment alternative for patients with NAFLD.

We could not find any study till date in the literature that investigate the effect of $\mathrm{NO}$ synthesis on liver and portal vein that is expected to increase in patients using vildagliptin. This study investigated the effect of the used type 2 DM drug, vildagliptin, a DPP- 4 inhibitor, on portal hemodynamics and hepatosteatosis.

\section{Methods}

\section{Patients}

This cross-sectional research was designed to evaluate two groups, each with 50 type $2 \mathrm{DM}$ cases, who were followed for at least 3 months and used the same drugs at the Outpatient Clinic. Patients were randomly assigned to treatment groups. The first group (Group 1) consisted of patients that used metformin (1000 mg bid) and gliclazide (60 mg qd). The second group (Group 2) consisted of patients that used vildagliptin (50 $\mathrm{mg}$ bid) in addition to the same amount of metformin and gliclazide since their glycated haemoglobin (HbA1c) was detected at $7 \%$ or higher. The patients were prospectively assigned to each of these two groups for the purpose of this study. Patients with type 2 DM older than 18 years that used metformin and gliclazide or metformin, gliclazide and vildagliptin for at least 3 months were included in the study. Patients who have diseases that may affect the portal vein pressure such as chronic liver disease, chronic renal failure, active infection and patients using certain drugs which may affect portal pressure such as propronalol, calcium channel blockers, angiotensin-converting enzyme inhibitors, angiotensin receptor blockers and isosorbit monohydrate were excluded from the study. Patients with body mass index (BMI) over $40 \mathrm{~kg} / \mathrm{m}^{2}$ and that used alcohol and cigarettes were also excluded from and recorded along with arterial blood pressure, height, and weight. Also low-density lipoprotein cholesterol (LDL-C), high-density lipoprotein cholesterol (HDL-C), triglyceride, and $\mathrm{HbA1c}$ levels were measured. Patients were questioned for history of other known diseases, operations, and use of other pharmaceutical drugs.

This study protocol was in accordance with the declaration of Helsinki and was approved by the Ethics Committee of Bezmialem Vakıf University. Written informed consent was obtained from each participant before commencement of the study (ClinicalTrials. gov Identifier: NCT01963130).

\section{Echo-Doppler Ultrasound}

Patients were examined in the left decubitus position with a Logiq 9 Review (GE, Milwaukee, WI, USA) ultrasound device and a $3.5-\mathrm{mHz}$ convex transducer probe was used. Gray scale and color Doppler features were used. First, all segments of the liver were examined and the presence and degree of hepatosteatosis was recorded. Next portal vein measurements were made at the level of the portal confluence. Doppler angle was maintained at $30^{\circ}-60^{\circ}$. Doppler gain and filter settings were adjusted. During the mid-inspiratory phase, the spectrum of portal vein was recorded for at least 5 seconds and measurements were performed through this wave pattern. Portal vein diameter, flow pattern, flow velocity, and flow rate were evaluated. Measurements were repeated three times and the average of these three measurements was recorded (9).

Blood samples were drawn after 12 hours of fasting in the morning hours, i.e., between 8:00 and 9:00 a.m., in the laboratory of Bezmialem Vakif University Hospital. Lipid profile was measured by chemiluminescent immunoassay method, using"Beckman Coulter" device. Glycated haemoglobin (HbA1c) levels were measured by turbidimetric inhibition immunoassay (Roche Diagnostics GmbH, Mannheim, Germany).

\section{Statistical analysis}

Statistical analyzes of data were performed using the Statistical Package for Social Sciences for Windows 13.0 (SPSS Inc., Chicago, IL, USA). Mean, median, and standard deviation were used for descriptive statistical evaluation where appropriate; t-tests compared normally distributed parameters. Mann-Whitney U test was used to compare non-normal distributed parameters, and Chi-square test was used to compare proportional data. Two-sided $\mathrm{p}$ value $<0.05$ was considered significant.

\section{Results}

A total of 97 patients completed the study. Three patients were dropped from the study because they did not accept examination by Doppler ultrasound. The number of enrolled cases in the study reduced to 49 cases with type $2 \mathrm{DM}$ in Group 1 (20 men, 29 women) and 48 cases in Group 2 (20 men, 28 women). Ages ranged from 35 to 79 years old. No 


\begin{tabular}{|c|c|c|c|}
\hline & Group $1(n=49)$ & Group $2(n=48)$ & $\mathbf{p}$ \\
\hline Age (years) & $57 \pm 9.9$ & $54.7 \pm 9.5$ & 0.26 \\
\hline $\mathrm{BMI}\left(\mathrm{kg} / \mathrm{m}^{2}\right)$ & $30.1 \pm 5.3$ & $31.1 \pm 6.8$ & 0.67 \\
\hline $\mathrm{HbA} 1 \mathrm{c}(\mathrm{mmol} / \mathrm{mol})$ & $54 \pm 12$ & $55 \pm 9$ & 0.53 \\
\hline HbA1c (\%) & $7.07 \pm 1.07$ & $7.23 \pm 1.3$ & \\
\hline MAP $(\mathrm{mmHg})$ & $120 \pm 16.4$ & $125 \pm 12.3$ & 0.62 \\
\hline LDL-C (mg/dL) & $135 \pm 36$ & $133 \pm 23$ & 0.9 \\
\hline Triglyceride (mg/dL) & $206 \pm 16.8$ & $192 \pm 24.4$ & 0.58 \\
\hline $\mathrm{HDL}-\mathrm{C}(\mathrm{mg} / \mathrm{dL})$ & $28 \pm 12.6$ & $32 \pm 14.2$ & 0.55 \\
\hline
\end{tabular}

Group 1: Cases who use metformin $(2 \times 1000 \mathrm{mg})$ and gliclazide $(1 \times 60 \mathrm{mg})$, Group 2: Cases who use metformin $(2 \times 1000 \mathrm{mg})$, gliclazide $(1 \times 60 \mathrm{mg})$ and vildagliptin (2×50 mg), MAP: mean arterial pressure; LDL-C: low-density lipoprotein cholesterol; HDL-C: high-density lipoprotein cholesterol.

Table 2. Portal vein flow velocity, portal vein flow, and portal vein diameter by group

\begin{tabular}{|c|c|c|c|}
\hline & Group $1(n=49)$ & Group $2(n=48)$ & $\mathbf{p}$ \\
\hline $\begin{array}{l}\text { Portal vein flow } \\
\text { velocity }(\mathrm{cm} / \mathrm{s})\end{array}$ & $7.6 \pm 1.6$ & $7.8 \pm 2.9$ & 0.6 \\
\hline $\begin{array}{l}\text { Portal vein flow } \\
\text { volume (mL/min) }\end{array}$ & $482.4 \pm 14$ & $478.5 \pm 23$ & 0.92 \\
\hline $\begin{array}{l}\text { Portal vein diameter } \\
(\mathrm{mm})\end{array}$ & $11.47 \pm 1.6$ & $11.43 \pm 1.8$ & 0.92 \\
\hline \multicolumn{4}{|c|}{$\begin{array}{l}\text { Group 1: Cases who use metformin }(2 \times 1000 \mathrm{mg}) \text { and gliclazide }(1 \times 60 \mathrm{mg}) \text {, } \\
\text { Group 2: Cases who use metformin }(2 \times 1000 \mathrm{mg}) \text {, gliclazide }(1 \times 60 \mathrm{mg}) \text { and } \\
\text { vildagliptin }(2 \times 50 \mathrm{mg}) \text {. }\end{array}$} \\
\hline
\end{tabular}

Table 3. The comparison of hepatosteatosis sign and degree of hepatosteatosis by group

\begin{tabular}{|l|c|c|c|}
\hline Stage & Group $1(\mathbf{n}=\mathbf{4 9})$ & Group 2 (n=48) & p \\
\hline Stage 0 & $8(16.6)$ & $14(29.2)$ & 0.13 \\
\hline Stage 1 & $20(40.8)$ & $18(37.5)$ & 0.45 \\
\hline Stages 2-3 & $21(42.9)$ & $16(33.3)$ & 0.33 \\
\hline
\end{tabular}

Group 1: Cases who use metformin $(2 \times 1000 \mathrm{mg})$ and gliclazide $(1 \times 60 \mathrm{mg})$, Group 2: Cases who use metformin (2x1000 mg), gliclazide (1×60 mg), and vildagliptin $(2 \times 50 \mathrm{mg})$.

significant difference was found in term of age, gender, BMI, HbA1c, mean arterial pressure, LDL-C, HDL-C or triglyceride levels in two groups. Table 1 shows the age of the patients and parameters of metabolic syndrome.

Portal vein flow velocity, portal vein flow, and portal vein diameter of all cases were measured by Doppler ultrasound in both groups. No significant difference was found between the groups (Table 2). There was no significant difference between groups regarding to ultrasonographic grading of hepatosteatosis (Table 3).
The duration of Group 2 vildagliptin use was $7.8 \pm 4.65$ (range 3-17 months) months. Seven patients and nine patients were using atorvastatin in group 1 and group 2 respectively.

\section{Discussion}

It is known that hepatosteatosis accompanied in the majority of patients with type 2 diabetes mellitus. In some cases with hepatosteatosis, developed steatohepatitis characterized by elevated liver enzymes and liver inflammation. Steatohepatitis is considered to be important in the etiology of the disease classified as cryptogenic liver cirrhosis (10). Another point is the release of $\mathrm{NO}$ which is an important vasodilator. $\mathrm{NO}$ is secreted mostly from endothelial cells and smooth muscles. It causes vasodilatation in many vessels as well as in the portal vein. $\mathrm{NO}$ also shows effects for the prevention of cirrhosis by reducing sinusoidal resistance, antifibrosis and antithrombosis. However, when over-secreted in patients with cirrhosis, it contributes to the hyperdynamic circulation and portal hypertension by means of vasodilatation and increased portal blood flow. At the same time, NO improves the growth of collateral artheries and causes collateral blood flow (6-8). This can contribute to variceal bleeding, one of the most feared complication in cirrhotic patients. Vildagliptin, used in the treatment of type 2DM, may lead to an increase in NO release by increasing incretins (11). In one study after icretin was given to the canine cell culture, it was found that NO levels in the portal vein increased (5). Another study demonstrated decreased levels of serum acetyl di-methyl arginine, which is recognized as an indirect indictor of $\mathrm{NO}$ elevations, in subjects receiving vildagliptin (12). In one another study that measured aortic and glomerular NO levels in obese rats using saxagliptin, showed that enhanced glycemic control with DPP4 inhibition improved NO release (13). In our study we aimed to determine the effects of increased NO levels on portal vein pressure and hepatic steatosis in patients with type $2 \mathrm{DM}$ using vildagliptin for longer than three months.

In our study, the two groups exhibited no difference in terms of parameters of metabolic syndrome. We could not find any effect of vildagliptin on portal venous flow, portal vein diameter, or flow rate. It could be that in treatment of diabetes, the process required for vildagliptin to exhibit a positive effect on hepatosteatosis and hemodynamics of the portal vein may require a longer period of time than the 3 months of this study period. When we evaluated the results, NO secretion is expected to increase in diabetics using vildagliptin. However, our study did not confirm a reduction in portal vein pressure by doppler ultrasound. This situation can be interpreted in three ways. Firstly, DPP-4 inhibitors increase NO release, but decrease the release of glucagon. While the release of NO cause vasodilatation in portal vein, on the contrary decreased glucagon levels cause vasoconstriction in the portal vein (14, 15). As a result, the result is meaningless because vildagliptin may have multiple effects via different mechanisms. Secondly, the majority of patients with type $2 \mathrm{DM}$ are known to have 
metabolic syndrome. It is thought that the release of NO decreases depending on the endothelial dysfunction in patients with metabolic syndrome $(7,8)$. As a result, the result is meaningless because most of these patients have metabolic syndrome and this does not increase the release of NO. Finally, even if the use of vildagliptin increase the release of NO in portal vein, this increase may not be sufficient to make changes in portal pressure.

Invasive angiographic examination is the gold standard for the measurement of hepatic venous pressure gradient and portal vein pressure; however, abdominal Doppler ultrasound is non-invasive and cheap, particularly for evaluation of patients with hepatic dysfunction. Doppler ultrasound is therefore a major diagnostic tool for noninvasive evaluation of hepatic vascular hemodynamics (16-18). In a study including 375 patients with portal hypertension, the sensitivity and specificity of parameters of portal vein by Doppler ultrasonography for demonstration of portal hypertension were $80 \%$ and $80 \%$, with a weak correlation between Doppler ultrasound findings and portal pressure. There was also a correlation between Doppler ultrasound findings and the severity of portal hypertension until occurrence of collaterals (17). In our study, no case had clinical or laboratory findings that support the development of collaterals; therefore, we may suggest Doppler USG as an appropriate, non-invasive method for assessment of portal vein pressure.

Patients who used vildagliptin treatment did not differ significantly with regards to hepatosteatosis grade. In the control group, there were no significant differences in hepatosteatosis or serum ALT levels. A published review on the effects of DPP-4s on the liver included studies which report that DPP-4 inhibitors corrected hepaticsteatosis as well as those which described a close association with hepaticsteatosis (19). A study investigating the effects of sitagliptin, a DPP-4 inhibitor, in patients with moderate hepatic impairment found that the drug was safe and did not cause clinical deterioration (20). Our results did not indicate a significant increase in hepaticsteatosis.

\section{Study limitations}

Since in our study exclusion criteria is kept wide to reduce the risks that affect portal pressure, the number of patients is limited. Declaration of patients and their relatives were taken into account since levels of GLP-1 in plasma cannot be measured. Therefore, there was not an objective criterion that shows if the patients use the drug or not. We could measure the level of portal pressure and hepatic steatosis before vildagliptin and 3 months after initiation of the drug. Threemonth period may be considered insufficient to detect the effect of vildagliptin on portal pressure by Doppler imaging. But by our cross sectional study, patients using vildagliptin for an average of 7 months enrolled in the study. Another point is that there was no study showing how sensitive Doppler ultrasound is to demonstrate the short-term change in portal flow.

\section{Conclusion}

A treatment of type $2 \mathrm{DM}$ patients with the incretin vildagliptin for at least 3 months had no effect on portal vein hemodynamics as assessed with Doppler ultrasound. Further long-term studies with better evaluation methods are needed to demonstrate the expected potential beneficial effect of vildagliptin on portal hemodynamics and hepatosteatosis.

Ethics Committee Approval: Ethics committee approval was received for this study from the Ethics Committee of Bezmialem Vakif University (07.05.2014 Decision No: 8/1 Issue: 7130664/020-0104/111).

Informed Consent: Written informed consent was obtained from all the patients who participated in this study.

Peer-review: Externally peer-reviewed.

Author Contributions: Concept - C.K., S.Y.; Design - C.K., O.K., R.K.; Supervision - M.Ç., M.K.; Resource - M.Z., R.E., M.A.Ç.; Materials - C.K., T.Ö., M.Ç., M.Z., S.A.; Data Collection and/or Processing - C.K., R.K., S.A.; Analysis and/or Interpretation - C.K., M.A.Ç.; Literature Search - C.K.; Writing - C.K.; Critical Reviews - S.Y.

Conflict of Interest: No conflict of interest was declared by the authors.

Financial Disclosure: The authors declared that this study has received no financial support.

\section{References}

1. Kim W, Egan JM. The Role of Incretins in Glucose Homeostasis and Diabetes Treatment. Pharmacol Rev 2008; 60: 470512. [CrossRef]

2. Deacon CF, Wamberg S, Bie P, Hughes TE, Holst JJ. Preservation of active incretin hormones by inhibition of dipeptidyl peptidase IV suppresses meal-induced incretin secretion in dogs. J Endocrinol 2002; 172: 355-62. [CrossRef]

3. Meier JJ, Goetze O, Anstipp J, Hagemann D, Holst JJ, Schmidt WE, et al. Gastric inhibitory polypeptide does not inhibit gastric emptying in humans. Am J Physiol Endocrinol Metab 2004; 286: E621-25.

4. Baggio LL, Huang Q, Cao X, Drucker DJ. An albumin-exendin- 4 conjugate engages central and peripheral circuits regulating murine energy and glucose homeostasis. Gastroenterology 2008; 134: 1137-47. [CrossRef]

5. Ding KH, Zhong Q, Xu J, Isales CM. Glucose-dependent insulinotropic peptide: differential effects on hepatic artery vs. portal vein endothelial cells. Am J Physiol Endocrinol Metab 2004; 286: 773-9. [CrossRef]

6. Alemany CA, Oh W, Stonestreet BS. Effects of nitric oxide synthesis inhibition on mesenteric perfusion in young pigs. Am J Physiol 1997; 272: 612-6. [CrossRef]

7. Angelico F, Del Ben M, Conti R, Francioso S, Feole K, Fiorello $\mathrm{S}$, et al. Insulin resistance, the metabolic syndrome, and nonalcoholic fatty liver disease. J Clin Endocrinol Metab 2005; 90: 1578-82. [CrossRef]

8. Wanless IR, Shiota K. The pathogenesis of nonalcoholic steatohepatitis and other fatty liver diseases: a four-step model including the role of lipid release and hepatic venular obstruc- 
tion in the progression to cirrhosis. Semin Liver Dis 2004; 24: 99-106. [CrossRef]

9. Patriquin H, Lafortune M, Burns PN, Dauzat M. Duplex Doppler examination in portal hypertension: technique and anatomy. Am J Roentgenol 1987; 149: 71-6. [CrossRef]

10. Cozma A, Orăşan O, Sâmpelean D, Fodor A, Vlad C, Negrean $\mathrm{V}$, et al. Endothelial dysfunction in metabolic syndrome. Rom J Intern Med 2009; 47: 133-40.

11. Ulusan S, Yakar T, Koc Z. Evaluation of portal venous velocity with doppler ultrasound in patients with nonalcoholic fatty liver disease. Korean J Radiol 2011; 12: 450-5. [CrossRef]

12. Cakirca M, Karatoprak C, Zorlu M, Kiskac M, Kanat M, Cikrikcioglu MA, et al. Effect of vildagliptin add-on treatment to metformin on plasma asymmetric dimethylarginine in type 2 diabetes mellitus patients. Drug Des Devel Ther 2014; 14: 239-43.

13. Mason RP, Jacob RF, Kubant R, Walter MF, Bellamine A, Jacoby A, et al. Effect of Enhanced Glycemic Control with Saxagliptin on Endothelial Nitric Oxide Release and CD40 Levels in Obese Rats. J Atheroscler Thromb 2011; 18: 774-83. [CrossRef]

14. Wiest R, Gronsmann R. Nitric oxid and portal hypertension: it's role in the regulation of intrahepatic and splanchnic vascular resistance. Semin Liver Dis 1999: 19: 411-26.
15. Hülagü S, Sentürk O, Erdem A, Ozgür O, Celebi A, Karakaya AT, et al. Effects of losartan, somatostatin and losartan plus somatostatin on portal hemodynamics and renal functions in cirrhosis. Hepatogastroenterology 2002; 49: 783-7.

16. Balci A, Karazincir S, Sumbas H, Oter Y, Egilmez E, Inandi T. Effects of Diffuse Fatty Infiltration of the Liver on Portal Vein Flow Hemodynamics. J Clin Ultrasound 2008; 36: 134-40. [CrossRef]

17. Berzigotti A, Gilabert R, Abraldes JG, Nicolau C, Bru C, Bosch J, et al. Noninvasive prediction of clinically significant portal hypertension and esophageal varices in patients with compensated liver cirrhosis. Am J Gastroenterol 2008; 103 : 1159-67. [CrossRef]

18. Haag K, Rössle M, Ochs A, Huber M, Siegerstetter V, Olschewski M, et al. Correlation of Duplex Sonography Findings and Portal Pressure in 375 Patients with Portal Hypertension. Am J Roentgenol 1999; 172: 631-5. [CrossRef]

19. Itou M, Kawaguchi T, Taniguchi E, Sata M. Dipeptidyl peptidase-4: a key player in chronic liver disease. World J Gastroenterol 2013; 19: 2298-306. [CrossRef]

20. Migoya EM, Stevens CH, Bergman AJ, Luo WL, Lasseter KC, Stacy $\mathrm{C}$, et al. Effect of moderate hepatic insufficiency on the pharmacokinetics of sitagliptin. Can J Clin Pharmacol 2009; 16: 165-70. 